\title{
Expression Profile of Micrornas (106b-3p, 130b-3, 145-3p and 199a-5p) in Urine and Serum Samples From Patients With the Diagnosis of Bladder Cancer
}

Piotr Kutwin ( $\nabla$ kutwin1986@gmail.com )

Medical University of Lodz: Uniwersytet Medyczny w Lodzi https://orcid.org/0000-0002-6681-3982

Edyta Marta Borkowska

Medical University of Lodz: Uniwersytet Medyczny w Lodzi

Paulina Bogucka

Medical University of Lodz: Uniwersytet Medyczny w Lodzi

Zbigniew Jablonowski

Medical University of Lodz: Uniwersytet Medyczny w Lodzi

Research

Keywords: RNAs, miRNA, PCR, cancer

Posted Date: October 8th, 2020

DOI: https://doi.org/10.21203/rs.3.rs-86217/v1

License: @ (i) This work is licensed under a Creative Commons Attribution 4.0 International License. Read Full License 


\section{Abstract}

Background MicroRNAs (miRNA) are short, single stranded, non-coding RNAs that play an important role in controlling gene expression at the post-transcriptional stage. There is no bladder cancer marker that has been approved as an alternative for diagnostic cystoscopy and urine cytology so far, thus research for alternative, more sensitive, and less invasive methods of bladder cancer detection are being made. The aim of the study was to compare the relative expression levels of miRNAs in patients with bladder cancer.

Materials and methods Urine and serum samples were collected from patients with the diagnosis of bladder cancer (NMIBC 71\%, MIBC 29\%). We assessed expression of 4 miRNAs (106b-3p, 130b-3, 145-3p and 199a-5p) using real-time PCR and double delta $(\triangle \Delta \mathrm{Ct})$ method. The analysis was performed with the Mann-Whitney $U$ test.

Results miRNA 145-3p was significantly underexpressed in urine $(p=0,0111)$ comparing with control group, whereas in serum we did not find relevant differences between groups ( $p=0,0903)$. Overexpression was observed for miRNA 199a$5 p$ tested in urine $(p=0,0262)$ and for miRNA 106b-3p for both urine and serum $(p=0,0262$ and $p=0,0149$ respectively) . For miR-130b-3 we did not find statistically significant differences neither for urine $(p=0,6335)$ nor serum $(p=0,2443)$.

Conclusions A correlation between the relative levels of expression for miRNA 106b-3p, 199a-5p and miRNA 145-3p was detected. We also observed differences between the results obtained for urine and serum. In the content of urinary cancers diagnosis urine seems to be more useful material than serum. We plan to continue our studies assessing expression levels of miRNA 106b-3.

\section{Background}

Bladder cancer ( $\mathrm{BCa}$ ) is ranked as the 9th most common cause of morbidity, and 13th cause of cancer deaths in the world. The highest incidence rates are observed in men in Southern and Western Europe and North America. [1] It is generally assumed that $75-85 \%$ of newly-diagnosed BCa belong to the NMIBC (non-muscle invasive bladder cancer) group, and in most cases, can be treated by TURBT (transurethral resection of the bladder tumor).[2] NMIBC is characterized by a high recurrence rate and risk of progression to cancer infiltrating the muscle of the bladder (MIBC) or by progression from a low-grade to a high-grade tumor.[3] Because of these features, there is a need to perform systematic follow-up cystoscopies, many of which show no recurrence of bladder tumors. Moreover, many endoscopic bladder inspections are made as a consequence of nonspecific symptoms commonly linked to BCa, given that fewer than $20 \%$ of patients presenting with hematuria actually suffer from urinary trancy malignancy.[4]

As the result, management in bladder cancer accounted for $3 \%$ of all cancer costs in the European Union, resulting in BCa becoming the most expensive malignancy. $[5,6]$ At present, cystoscopy supported by urine cytology is the standard approach in the diagnosis and follow-up of BCa.[7] The development of a comprehensive bladder cancer biomarker would significantly improve the quality of care for patients suffering from this disease and reduce costs associated with the diagnosis and follow-up. However, distinct molecular pathways in the development of low and high grade tumors raise challenges as many biomarkers cannot be used in detection of both types of cancer.[8] Urine cytology is a noninvasive test for BCa with very high specificity. However, it has low sensitivity, particularly in detecting low grade tumors. [9] To date, none of the bladder cancer markers, including urine cytology, have been approved as an alternative for diagnostic cystoscopy.

The unsatisfactory sensitivity and specificity of available cancer markers has mandated a search for alternative diagnostic approaches. The discovery of small non-coding RNA is considered as an opportunity to better understand cancer biology. miRNAs, which consist of 19-22 nucleotides, regulate gene expression in such processes as angiogenesis, cell proliferation and apoptosis. $[10,11]$ It has been shown that some miRNAs are dysregulated in cancer 
cells.[12] MiRNA are stable in the extracellular form in body fluids (blood, urine, saliva, peritoneal fluid).[13] miRNA stabilized by exosomes are less susceptible to degradation than long polyadenylated mRNA.[14] Given these two reasons, $\mathrm{BCa}$ offers a good target-cancer for further examination of miRNA as a potential cancer biomarker.

Extracellular vesicles (EVs) are small membranous vesicles which are involved in communication between cells by transferring molecules (including miRNA). EVs take a role in the regulation of physiological, as well as pathophysiological processes such as tumorigenesis. miRNAs transported by EVs target a recipient cell influencing changes in its biology.[15] miRNA in EVs are considered a good marker as they are further protected against rapid degradation by a 2-layer lipid membrane covering EVs.[16] In order to obtain miRNA associated with EVs urine must be centrifuged and urine supernatant should be used for extraction of nucleic acids.

We have chosen miRNA 106b-3p, 130b-3, 145-3p and 199a-5p as their expression have been proven to be altered in patients diagnosed with cancers. Interactions between circSLC8A1 and miRNA 130b targeting gene PTEN, which plays a role in suppression of bladder cancer, were studied by Lu et al.[17] Research performed by Jiang on patients with BCa studied the regulatory mechanisms between miRNA 106 and baicalein which has an anti-tumor role.[18] miRNA 106b-5p could be used as prognostic marker differentiating between more and less-favourable prognostic groups of BCa in which TP53 mutation was found.[19] Data obtained by Ahmadi et al. suggest a causative role for miRNA 199a-5p in the tumorigenesis of lung as well as several other potential cancers.[20] Retrospective studies of bladder cancer tissues

performed by Ecke et al. have shown that miRNA 199a-3p can be used as an independent marker of overall survival.[21] Sun et al. have studied mechanisms by which miRNA 145-5p suppresses cell growth and migration ability in BCa.[22] As our meta-analysis have showed combining many miRNAs into one test has better sensitivity in detecting patients with bladder cancer compared to tests based on only single miRNa.[23

The aim of the study was to compare the relative expression levels of miRNAs 106b-3p, 130b-3, 199a-5p and 145-3p in serum and urinary supernatant in patients with bladder cancer.

\section{Materials And Methods}

The serum and urine samples were obtained from 17 patients ( 4 women and 13 men) admitted to the urology department. The age range was 38-84 years with an average age of 65.7 . The majority of the patients were male (76\%). Twelve patients $(71 \%)$ in the group were diagnosed with non-invasive bladder cancer, and the remaining 5 patients $(29 \%)$ had invasive bladder cancer in stage pT2. None of the patients were diagnosed with CIS (carcinoma in situ). Tumor histological grade was determined according to the World Health Organization/International Society of Urological Pathology criteria of 2004, and tumor stage was assessed in accordance with the 2002 UICC TNM classification [24, 25]. The control group consisted of 3 samples isolated from oncologically healthy patients admitted to the urology ward. They underwent cystoscopy excluding tumor changes in the bladder.

Characteristics of tested population is shown in Table 1 (Table 1). 
Table 1

Characteristic of tested population

\begin{tabular}{|lll|}
\hline & & Results \\
\hline Gender & Male & $13(76 \%)$ \\
\hline Tumor grade & Female & $4(24 \%)$ \\
\hline & Low-grade & $10(59 \%)$ \\
\hline Tumor stage & High-grade & $7(41 \%)$ \\
\hline & pTa & $10(59 \%)$ \\
\hline Smoking history & pT1 & $2(12 \%)$ \\
\hline Occupational exposure & pT2 & $5(29 \%)$ \\
\hline Age [years] & & $5(29 \%)$ \\
\hline & minimum & 38 \\
\hline & maximum & 84 \\
\hline
\end{tabular}

The inclusion criteria for the study of the tested group comprised age over 18 years old, newly-diagnosed bladder cancer and the ability to give informed consent. The exclusion criterion was the history of bladder cancer in the past or recurrent bladder cancer and history of any other neoplasm. Inclusion criteria for control group were absence of current bladder tumor and no history of bladder cancer nor any other neoplasm.

The permission to conduct the study was granted by the Bioethics Advisory Commission at Lodz Medical University, No. RNN/231/17/KE, and all the patients signed consent forms. The study was supported by Medical University Grant No. 502-03/5-138-02/502-54-146.

Urine and blood samples were collected before TURBT, and were stored for less than four hours, at $+4{ }^{\circ} \mathrm{C}$, before miRNA isolation. Urine and blood samples were centrifuged for 15 minutes at $3000 \mathrm{rpm}$ and the resulting urine supernatant or plasma were stored at $-20^{\circ} \mathrm{C}$.

We applied the isolation protocol of TriPure Isolation Reagent, Roche, Cat No. 11667 165001, Version 07 which was used to isolate miRNAs. The amount and quality of isolated RNA was measured spectrophotometrically.[26] In addition, the quality of the obtained isolate was checked electrophoretically on a $2 \%$ agarose gel, which allowed for assessment of the degree of RNA degradation. Qualitative and quantitative assessment of DNA and RNA was made on the basis of spectrophotometric measurement of absorbance at $260 \mathrm{~nm}, 280 \mathrm{~nm}$, and $230 \mathrm{~nm}$ light length. The measurement was performed using the Implen NanoPhotometer NP80 device.

For real time PCR (Reverse Transcription Reaction), we used the protocol for High- Capacity cDNA Reverse Transcription Kits, Applied Biosystems, Cat No. 4368813, Lot No. 1201113. Random pairs of primers were used in the kit, ensuring synthesis efficiency for all RNA molecules present in the sample, including mRNA and rRNA. To assess the expression of individual microRNAs in the serum and urine supernatant, real-time PCR was performed with primers specific for miRNAs 106b-3p, 130b-3, 145-3p, and 199a-5p using the miRCURY LNA Universal RT microRNA PCR set (Exiqon. Cat No. 203351, T100 Thermal Cycler, Bio-Rad), following the manufacturer's protocol. Reactions were carried out in special 96- 
well plates on a BIO-RAD CFX 96 Real-Time System device. Each reaction was performed twice, and the arithmetic average of these two measurements was taken for further analysis.

Expression levels for the 106b-3p, 130b-3, 145-3p, 199a-5p microRNA were assessed using a comparative method, also known as the double delta $(\Delta \Delta \mathrm{Ct})$ method which is used to determine the relative difference in the expression level between tested samples and the reference. The first stage consists of the analysis of the marked $\mathrm{Ct}$ (the cycle at which the fluorescence level reaches a certain amount) in the amplification reaction of the examined miRNAs and control miRNA for both the tested and the control groups.

$(\Delta \mathrm{Ct})($ tested group $)=\mathrm{Ct}$ miRNA target $-\mathrm{Ct}$ miRNA reference

$(\Delta \mathrm{Ct})($ control group $)=\mathrm{Ct}$ miRNA target - Ct miRNA reference

Next, $\Delta \Delta$ Ct was calculated for each sample:

$\Delta \Delta \mathrm{Ct}=\Delta \mathrm{Ct}($ tested samples $)-\Delta \mathrm{Ct}($ control group)

The calculation of the relative expression level $(R)$ of the tested marker in the tested sample against the control sample was made using a formula: $\mathrm{R}=2^{-\Delta \Delta \mathrm{Ct}}$

The $\Delta \Delta$ Ct method assumes a uniform PCR amplification efficiency of $100 \%$ across all samples. In our study, the eciency was between $98.9 \%$ and $100 \%$. The value of $R=1$ means that the level of microRNA expression in the test is the same as in the calibration sample. A value of $\mathrm{R}>1$ indicates that the level of expression in the test sample is higher than the calibration sample. A value $\mathrm{R}<1$ means lower expression level in the test sample than in the calibration sample. $[27,28]$

The program PQStat version 1.6.8.304. was used to make statistical calculations. The analysis was performed with the Mann-Whitney $U$ test. The value $p<0.05$ was accepted as the threshold of statistical difference.

\section{Results}

The statistical analysis indicated that miRNA $145-3 p$ is significantly underexpressed in urine $(p=0,0111)$. For miRNA $145-3 p$ assessed in serum we did not find relevant differences between groups $(p=0,0903)$. Overexpression was observed for miRNA 199a-5p tested in urine $(p=0,0262)$ and for miRNA 106b-3p for both urine and serum, $p=0,0262$ and $p=0,0149$, respectively. For miR-130b-3 we did not find statistically significant differences nor for urine $(p=0,6335)$ nor serum $(p=0,2443)$. (Table 2, Fig. 1) 
Table 2

Relative expression levels of miRNAs (106b-3p, 130b-3, 145-3p and 199a-5p)

\begin{tabular}{|c|c|c|c|c|c|c|}
\hline \multicolumn{7}{|c|}{ miRNA expression (R) } \\
\hline miRNA & Sample & Median [n = 17] & IQR & Minimum & Maximum & Mann-Whitney U test \\
\hline \multirow[t]{2}{*}{$106 b-3 p$} & serum & 4.75 & 7.48 & 0.9 & 14.54 & $p=0.0262$ \\
\hline & urine & 18.57 & 19.425 & 1.19 & 33.33 & $p=0.0149$ \\
\hline \multirow[t]{2}{*}{$130 b-3$} & serum & 0.24 & 0.335 & 0.01 & 1.26 & $p=0.2443$ \\
\hline & urine & 2.78 & 3.475 & 0.44 & 6.42 & $p=0.6335$ \\
\hline \multirow[t]{2}{*}{$199 a-5 p$} & serum & 1.23 & 1.015 & 0.69 & 2.61 & $p=0.0903$ \\
\hline & urine & 1.46 & 1.145 & 0.94 & 2.62 & $p=0.0262$ \\
\hline \multirow[t]{2}{*}{$145-3 p$} & serum & 0.76 & 0.33 & 0.34 & 0.99 & $p=0.0903$ \\
\hline & urine & 0.36 & 0.255 & 0.14 & 0.95 & $p=0.0111$ \\
\hline \multicolumn{7}{|c|}{ statistically significant differences $(p<0.05)$ are bolded } \\
\hline \multicolumn{7}{|c|}{$\mathrm{n}$-number of patients } \\
\hline \multicolumn{7}{|c|}{ IQR -interquartile range } \\
\hline
\end{tabular}

We did not find link between miRNA expression and age, sex, status of smoking, or occupational involvement $(p>0.05)$. For tested miRNAs, there was no link between either an increase or decrease in the relative expression level and the tumor histological grade and stage $(p>0.05)$.

\section{Discussion}

The discovery of microRNA took place in 1993. The first species in which the short non-coding RNAs chains were found was nematode Caenorhabditis elegans.[29] In 2007, the first mention of miRNA alteration occurring in BCa was published.[30] Drawing on experience from other investigators, we have created an original diagnostic plate consisting of miRNAs that have been shown to be altered in cancer patients. In recent years, interest in miRNAs has increased significantly with research focusing on their role in bladder cancer. Homami A. and Ghazi F. in their review describe that miRNAs can act as both oncogenes and tumor suppressors. In addition, different types of cancers, cancer stages, and degrees of differentiation may have unique miRNA expression profiles. Thus, these molecules offer potential biomarkers for diagnosing bladder cancer.[31]

Detection of single miRNAs in urine samples is no more effective than current cytological tests due to the relatively low diagnostic specificity of such analyses.[32] Recent studies have used multiple miRNA panels to improve the diagnostic specificity of miRNA detection. It is important to select appropriate miRNA combinations and validate them by conducting outpatient testing using such panels. Although specificity is in favor of multi-miRNA, compared to singlemiRNA tests, it is still lower than the specificity of urine cytology.[23]

Our observations regarding the relative expression profile of miRNA 106b coincide with those made by Zhou X. et al. have also observed higher miRNA 106b expression in urine supernatant collected from patients with BCa, and additionally lowering of it's relative levels following tumor resection. In their study, they also present a case in which a patient showing relapse of a bladder tumor was associated with recurrence of high miRNA 106b expression.[33] 
Concerning miRNA 199a-5p Sakaguchi et al. have shown that the miRNA 199a family acts as tumor suppressors by regulating integrin alpha-3 (ITGA3). According to our study, miRNA 199a-5p was underexpressed in bladder cancer tissues.[34] Low expression of miRNA 199a-5p was also observed by Zhou M. et al. and T. Song in bladder cancer tissues specimens.[35, 36] These observations are in line with our results, as mild overexpression of miRNA 199a-5p was observed in the urine samples from all but one patient. While Zhou and Sakaguchi based their research on bladder cancer tissue, the present study examined the expression of miRNAs in blood serum and urine supernatant.

According to studies performed by Liu X. et al., overexpression of miRNA 130b stimulates proliferation, migration, and facilitates invasion of bladder cancer cells, while underexpression is associated with the opposite effect.[37] Although miRNA 130b seems to be upregulated in bladder cancer cells we did not find a correlation between blood serum and urine supernatant expression of miRNA $130 \mathrm{~b}-3$.

Zhang X. miRNA 145 inhibits migration and invasion of BCa cells by regulating N-cadherin. Studies have shown a reduced level of this miRNA in cells and tissues of patients with bladder cancer as compared to the control group.[38] Similar observations were noted in our study, however, on other biological material. We also found that the relative expression levels of miRNA 145-3p in urine of patients suffering from BCa was reduced. Such a correlation between tissue and urine miRNA expression levels is important as it shows that results of the studies made on histopathological material can be used in research of cancer markers in physiological fluids.

Our analyses have some limitations. The most important is the small number of samples. However, these are only preliminary results to determine whether any correlations between these materials may be present. As our database of collected bladder cancer samples grows, it will be possible to perform more detailed analyses on larger groups. In the initial period of the study, we did not want to focus on a single subgroup of bladder cancer, e.g. low-grade, high-grade, NMIBC or MIBC. By taking this approach, our study was not affected by any bias that could result from patient selection; however, the studied group demonstrated greater heterogeneity, although this may be diminished as the group of patients enlarges.

Urine is a good material for analysis as it is relatively easy to collect and makes direct contact with bladder tumors. Markers in plasma samples are more diluted due to the greater total volume of blood and natural blood-tissue barriers. We have also found that carrying out such studies in cooperation between two departments, sometimes located a few kilometers apart, to be a challenging task, as the material requires centrifugation and correct storage within a few hours after sample collection to avoid tissue degradation.

\section{Conclusions}

A correlation between the relative levels of expression in urine for miRNA 106b-3p, 199a-5p (an increase), and miRNA $145-3 p$ (a decrease), was observed. Preliminary results show that optimization of the collection and transport of biological material for genetic testing is a prerequisite for obtaining reliable results for future personalization of diagnosis and treatment. We generally received higher relative expression levels for miRNAs assessed in urine compared to serum. For serum statistically significant differences were observed only for miRNA 106b-3p, and they corresponded to higher expression levels of miRNA 106b-3p in urine. In the context of urinary cancers diagnosis urine seems to be more useful material than serum. We plan to continue our studies assessing expression levels of miRNA 106b-3p.

\section{List Of Abbreviations}

BCa -Bladder cancer

TURBT -transurethral resection of bladder tumor 
NMIBC -non-muscle invasive bladder cancer

MIBC -muscle invasive bladder cancer

miRNA -microRNA

EVs -Extracellular vesicles

\section{Declarations}

\section{Ethics approval and consent to participate}

The permission to conduct the study was granted by the Bioethics Advisory Commission at Lodz Medical University, No. RNN/231/17/KE, and all the patients signed consent forms.

\section{Consent for publication}

Not applicable

\section{Availability of data and materials}

All available data is attached to supplementary materials

\section{Competing interests}

None

\section{Funding}

The study was supported by Medical University Grant No. 502-03/5-138-02/502-54-146.

\section{Authors' contributions}

PK preparing main manuscript, data collection, data analysis and interpretation

EB reviewing the main manuscript, data collection, data interpretation

PB data collection, material processing

ZJ reviewing the main manuscript, conceptualization

\section{Acknowledgements}

None

\section{References}

1. AntoniS,FerlayJ,SoerjomataramI,ZnaorA,JemalA,BrayF.Bladder cancer incidence and mortality: a global overview and recent trends. European urology,2017,71.1:96-108.

2. CompératE,LarréS,RoupretM,etal.Clinicopathological characteristics of urothelial bladder cancer in patients less than 40 years old.Virchows Arch.2015;466(5):589-594.doi:10.1007/s00428-015-1739-2 
3. PatschanO,SjödahIG,ChebilG,etal.A molecular pathologic framework for risk stratification of stage T1 urothelial carcinoma.European urology,2015,68.5:824-832.

4. Boorjian SA, Raman JD, Barocas

DA,EvaluationandManagementofHematuria.WeinAJ,KavoussiLR,PartinAW,PetersCA,Campbell-

WalshUrology,11thedn,Philadelphia:Saunders,2012;Vol.I,Chapt9,pp. 183-194.

5. LealJ,Luengo-FernandezR,SullivanR,WitjesJA.Economic burden of bladder cancer across the European Union.European urology,2016,69.3:438-447.

6. AvritscherEB,CooksleyCD,GrossmanHB,etal.Clinical model of lifetime cost of treating bladder cancer and associated complications.Urology,2006,68.3:549-553.

7. WitjesJA.Bladder carcinoma in situ in 2003: state of the art.European urology,2004,45.2:142-146.

8. CattoJW,MiahS,OwenHC,etal.Distinct microRNA alterations characterize high-and low-grade bladder cancer.Cancer research,2009,69.21:8472-8481.

9. PlanzB,JochimsE,DeixT,CaspersHP,JakseG,BoeckingA.The role of urinary cytology for detection of bladder cancer.European Journal of Surgical Oncology (EJSO),2005,31.3:304-308.

10. BartelDP.MicroRNAs: genomics, biogenesis, mechanism, and function.cell,2004,116.2:281-297.

11. Calin GA, Sevignani C, Dumitru CD,etal.HumanmicroRNAgenesarefrequentlylocatedatfragilesitesandgenomicregionsinvolvedincancers. Proceedings of the National Academy of Sciences,2004,101.9:2999-3004.

12. Esquela-KerscherA,SlackFJ.Oncomirs-microRNAs with a role in cancer.Nature reviews cancer,2006,6.4:259-269.

13. WeberJA,BaxterDH,ZhangS,etal.The microRNA spectrum in 12 body fluids.Clinical chemistry,2010,56.11:17331741.

14. ValadiH,EkströmK,BossiosA,SjöstrandM,LeeJJ,LötvallJO.Exosome-mediated transfer of mRNAs and microRNAs is a novel mechanism of genetic exchange between cells.Nature cell biology,2007,9.6:654-659.

15. SahebiR,LangariH,FathinezhadZ,etal.Exosomes: New insights into cancer mechanisms.Journal of cellular biochemistry,2020,121.1:7-16.

16. KogaY,YasunagaM,MoriyaY,etal.Exosome can prevent RNase from degrading microRNA in feces.Journal of gastrointestinal oncology,2011,2.4:215.

17. LuQ,LiuT,FengH,etal.Circular RNA circSLC8A1 acts as a sponge of miR-130b/miR-494 in suppressing bladder cancer progression via regulating PTEN.Molecular cancer,2019,18.1:111.

18. JiangL,SongH,GuoH,WangC,LuZ.etal.Baicalein inhibits proliferation and migration of bladder cancer cell line T24 by down-regulation of microRNA-106.Biomedicine \& Pharmacotherapy,2018,107:1583-1590.

19. LeeE,Collazo-LorduyA,Castillo-MartinM,etal.Identification of microR-106b as a prognostic biomarker of p53-like bladder cancers by ActMiR.Oncogene,2018,37.44:5858-5872.

20. AhmadiA,KhansarinejadB,HosseinkhaniS,GhaneiM,MowlaSJ.miR-199a-5p and miR-495 target GRP78 within UPR pathway of lung cancer.Gene,2017,620:15-22.

21. EckeTH,StierK,WeickmannS,etal.miR-199a-3p and miR-214-3p improve the overall survival prediction of muscleinvasive bladder cancer patients after radical cystectomy.Cancer medicine,2017,6.10:2252-2262.

22. SunM,ZhaoW,ChenZ,etal.Circ_0058063 regulates CDK6 to promote bladder cancer progression by sponging miR145-5p.Journal of cellular physiology,2019,234.4:4812-4824.

23. KutwinP,KoneckiT,BorkowskaEM,Traczyk-BorszyńskaM,JabłonowskiZ.Urine miRNA as a potential biomarker for bladder cancer detection-a meta-analysis.Central European journal of urology,2018,71.2:177. 
24. Sobin LH, Gospodarowicz MK, Wittekind C.IUAC, editors. TNM Classification of Malignant Tumors. 6thed. Berlin/Heidelberg: Springer; 2002.

25. CompératEM,BurgerM,GonteroP,etal.Grading of Urothelial Carcinoma and The New "World Health Organisation Classification of Tumours of the Urinary System and Male Genital Organs 2016".Eur Urol Focus.2019;5(3):457-466.

26. Borkowska E, Kutwin P, Rolecka D, Konecki T, Borowiec M, Jabłonowski Z.Clinical value of microRNA-19a-3p and microRNA-99a-5p in bladder cancer.Archives of Medical Science,2019,15.1.

27. TyburskiJ,StudzińskaA,DacaP,TretynA.PCR w czasie rzeczywistym. Metody analizy danych.Biotechnologia,2008,1.80:86-96.

28. BorkowskaEM,KoneckiT,PietrusińskiM,BorowiecM,JabłonowskiZ.MicroRNAs Which Can Prognosticate Aggressiveness of Bladder Cancer.Cancers (Basel).2019;11(10):1551.Published 2019 Oct 14.

29. KutterC,SvobodaP.miRNA,siRNA, piRNA. Knowns of the unknown.RNA Biology,2008,5.4:181-188.

30. GottardoF,LiuCG,FerracinM,etal.Micro-RNA profiling in kidney and bladder cancers.Urol Oncol.2007;25(5):387-392.

31. HomamiA,GhaziF.MicroRNAs as biomarkers associated with bladder cancer.Medical journal of the Islamic Republic of Iran,2016,30:475.

32. EnokidaH,YoshinoH,MatsushitaR,NakagawaM.The role of microRNAs in bladder cancer.Investigative and clinical urology,2016,57.Suppl 1:S60-S76.

33. ZhouX,ZhangX,YangY,etal.Urinary cell-free microRNA-106b as a novel biomarker for detection of bladder cancer.Medical oncology,2014,31.10:197.

34. SakaguchiT,YoshinoH,YonemoriM,etal.Regulation of ITGA3 by the dual-stranded microRNA-199 family as a potential prognostic marker in bladder cancer.British journal of cancer,2017,116.8:1077-1087.

35. ZhouM,WangS,HuL,LiuF,ZhangQ,ZhangD.miR-199a-5p suppresses human bladder cancer cell metastasis by targeting CCR7.BMC urology,2016,16.1:64.

36. Song T, Zhang X, Yang G, Song Y, Cai W. Decrement of miR-199a-5p contributes to the tumorigenesis of bladder urothelial carcinoma by regulating MLK3/NF-KB pathway. Am J TransI Res. 2015;7(12):2786-94..Published 2015 Dec 15.

37. LiuX,KongC,ZhangZ.miR-130b promotes bladder cancer cell proliferation, migration and invasion by targeting VGLL4.Oncology reports,2018,39.5:2324-2332.

38. Zhang XF, Zhang XQ, Chang ZX, Wu CC, Guo H. microRNA-145 modulates migration and invasion of bladder cancer cells by targeting N-cadherin. Mol Med Rep. 2018;17(6):8450-6. doi:10.3892/mmr.2018.8910.

\section{Figures}



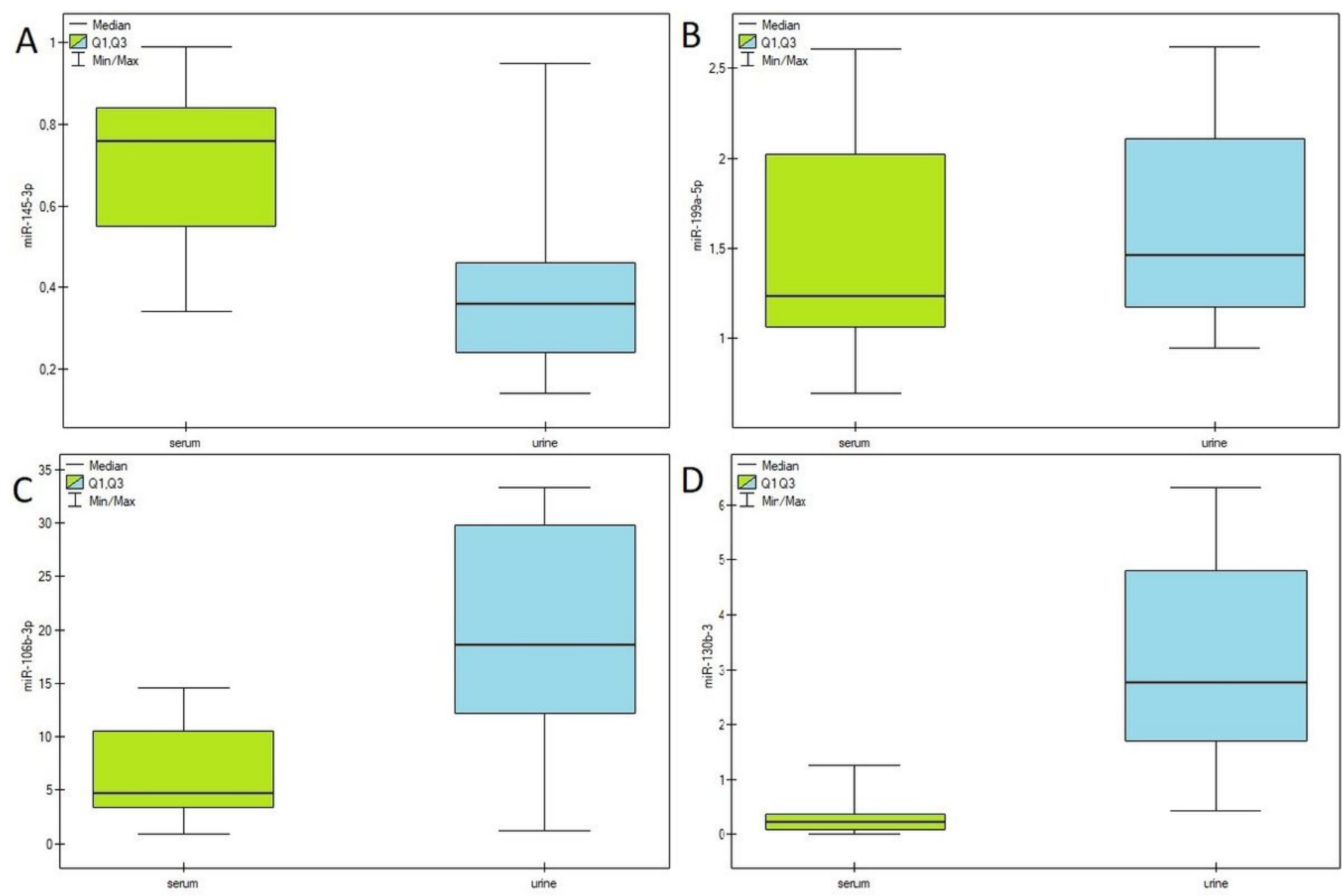

Figure 1

The statistical analysis indicated that miRNA $145-3 p$ is significantly underexpressed in urine $(p=0,0111)$. For miRNA $145-3 p$ assessed in serum we did not find relevant differences between groups $(p=0,0903)$. Overexpression was observed for miRNA 199a-5p tested in urine $(p=0,0262)$ and for miRNA 106b-3p for both urine and serum, $p=0,0262$ and $p=0,0149$, respectively. For miR-130b-3 we did not find statistically significant differences nor for urine $(p=0,6335)$ nor serum $(p=0,2443)$.

\section{Supplementary Files}

This is a list of supplementary files associated with this preprint. Click to download.

- supplementary130b.xlsx

- supplementary199a5p.xlsx

- supplementary106b3p.xlsx

- supplementary1453p.xIsx 